\title{
A grey-forecasting based multiple linear regression model for planning nursing homes
}

\author{
Tianyi $\mathrm{Xu}^{1, *}$, Sheng $\mathrm{Li}^{1}$ \\ ${ }^{1}$ School of Transportation Engineering, Nanjing Tech University, Nanjing, Jiangsu, China
}

\begin{abstract}
With the increasing demand for elderly care institutions in society, the issue of elderly care has become a serious social problem and a widely publicised livelihood issue. In order to actively respond to the trend of a deeply ageing population, the infrastructure of urban elderly care services is being strengthened. Led by relevant government departments, many scholars are exploring a model suitable for the development of elderly care in China, taking into account the experience of elderly care services at home and abroad. This paper proposes a universal planning model for elderly institutions based on multivariate integer linearity, introducing the zoning method and grey forecasting. It solves the problem of deciding the number of elderly institutions to be built in each district of a particular city in the government's future planning. Using Nanjing as an example, the model is then substituted with data from the Nanjing Rating Standards for Nursing Homes (for Trial Implementation) to obtain a table of construction plans for various types of nursing homes in five major stages during the period 2021-2035 and a map of the recommended distribution of nursing homes. The model simplifies complex calculations by transforming multivariate non-linear problems into linear ones. The simulation results have been proved to be practical and universal. The research results of the thesis can provide a theoretical basis and decision-making reference for the construction projects of elderly institutions and other functional infrastructures where the population gathers, which is conducive to the promotion of urbanisation and pulling economic growth, and provides material guarantee for the improvement of people's living standards.
\end{abstract}

\section{Introduction}

Under the background of the aging of the population, the social structure and patterns of China's society have undergone a major transformation, and China has become one of the fastest aging countries in the world. At the same time, demographically, the parents of the first generation of only children have entered old age and will successively enter a stage where they need support and care. Therefore, it is a national concern to find a solution for planning access to elderly care institutions in line with the national context and the people's situation. In line with the policy, in recent years, scholars in China have been conducting more comprehensive studies on the planning of elderly care institutions. Previously, scholars have combined the current situation of planning and design of county-level regional centers for the elderly, carried out rationalization analysis, and put forward the key points of architectural design for county-level regional centers for the elderly. The relevant literature covers three major levels: macro, meso, and micro [1]. However, due to the diversity of regional characteristics, most scholars' research results are only specific to particular counties and cities and are not universally applicable. Moreover, most scholars do not have a systematic theoretical framework to support them, and no scholars have yet summarized a standardized model for planning elderly institutions.
Against this background, it is of great practical significance to study the current situation of the utilization of elderly care institutions and the factors influencing them. It will help the government grasp the current situation of social elderly care services and improve the shortcomings of the existing service system.

\section{Planning model for nursing homes}

\subsection{Model for population forecast}

The number of elderly people is discrete data, which is relatively deterministic and difficult to predict and requires a dynamic model in differential equations.

The method of predicting this model can be the grey forecasting method. Grey forecasting is a method of predicting systems with uncertainty by identifying the degree of dissimilarity between the trends of aging population growth factors, i.e., correlation analysis, and generating raw population data to find the pattern of system changes. It can generate data series with strong regularity, and then build the corresponding differential equation model, to predict the future development trend [2].

The GM $(1,1)$ model is the basic model of grey forecasting theory. It does not require a large sample size,

\footnotetext{
* Corresponding author: Guanghua.ren@gecacademy.cn
} 
and there is no requirement for the distribution of the sample to be regular or not [3]. In data processing, the workload is relatively small, and the results of qualitative and quantitative analysis are usually close to each other. It is mostly used for medium and long-term forecasting and has a wide range of applications.

$$
\hat{x}^{(1)}(k+1)=\left(x^{(0)}(1)-\frac{b}{a}\right) e^{-a k}+\frac{b}{a}, k=0,1, \ldots, n-1, \ldots
$$

$$
\hat{x}^{(0)}(k+1)=\hat{x}^{(1)}(k+1)-\hat{x}^{(1)}(k), k=1,2, \ldots, n, \ldots
$$

Where $\mathrm{x}^{\wedge}((1))(k)$ denotes the new series after accumulation, $\mathrm{k}$ denotes the ordinal number of the data and $\mathrm{n}$ denotes the number of data

By analyzing this model, we can get the data of the future elderly population in each district of a city and then get the data of the density of the elderly population in each district of a city by density $=$ population/area.

\subsection{Model for planning nursing homes}

\subsubsection{Criterions}

2.2.1.1 Criterion 1: Integration. When considering the number of institutions to be built, a "zoning decision is made, and the arrangement is coordinated". The process of building institutions in each of the major districts will be arranged in an integrated manner.

2.2.1.2 Criterion 2: Scope. The number of institutions must be set within the area of the local area.

2.2.1.3 Reasonableness. As far as possible, the needs of the elderly population in different areas and at different consumption levels should be taken into account [4].

The main factors influencing the number of nursing homes are economic income, social humanities, and health care. With total cost as the dependent variable and the number of nursing homes and falsification in each district as the independent variables, multiple linear regression can be used to investigate the relationship between a dependent variable and multiple independent variables [5].

\subsubsection{Matrix analysis of variable}

The analysis of the influencing parameters shows that this development planning model is a multi-objective nonlinear integer programming. For the sake of the text convenience of the chapter narrative, the number of proposed aged care institutions within zone one is denoted as $A i$, zone two as $B i$, zone three as $C i$, and the fourth zone as $D i$, where the subscript $i$ denotes the rank of the institution. The variable matrix is then.

$$
\mathrm{X}^{*}=\left[\begin{array}{lllll}
\mathrm{A}_{1} & \mathrm{~A}_{2} & \mathrm{~A}_{3} & \mathrm{~A}_{4} & \mathrm{~A}_{5} \\
\mathrm{~B}_{1} & \mathrm{~B}_{2} & \mathrm{~B}_{3} & \mathrm{~B}_{4} & \mathrm{~B}_{5} \\
\mathrm{C}_{1} & \mathrm{C}_{2} & \mathrm{C}_{3} & \mathrm{C}_{4} & \mathrm{C}_{5} \\
\mathrm{D}_{1} & \mathrm{D}_{2} & \mathrm{D}_{3} & \mathrm{D}_{4} & \mathrm{D}_{5}
\end{array}\right], A_{i}, B_{i}, C_{i} 、 D_{i} \in
$$

\subsubsection{Constraint analysis}

\subsubsection{Economic income}

In the process of building elderly care institutions, a minimum number of institutions will be built, taking into account the needs of elderly people with low, lower, middle, upper middle, and high disposable incomes, to prevent the lack of a specific class of elderly care institutions due to competition in the market economy and to cater for the needs of various income groups [6]. The institutions will provide exclusive and customized services for such elderly people for the AAAA and AAAA categories. According to the National Bureau of Statistics, $5 \%$ of society's population holds wealth well above the average, and they tend to demand customized services in their residences.

$$
\begin{aligned}
& \frac{A_{4}+A_{5}+B_{4}+B_{5}+C_{4}+C_{5}+D_{4}+D_{5}}{\sum_{1}^{5} A_{i}+\sum_{1}^{5} B_{i}+\sum_{1}^{5} C_{i}+\sum_{1}^{5} D_{i}} \geq 5 \%, \\
& A_{i} \geq A_{\text {min }}, i=1,2,3,4,5 \\
& B_{i} \geq B_{\text {min }}, i=1,2,3,4,5 \\
& C_{i} \geq C_{\text {min }}, i=1,2,3,4,5 \\
& D_{i} \geq D_{\text {min }}, i=1,2,3,4,5
\end{aligned}
$$

\subsubsection{Economic income}

The central urban area has been influenced by favorable policies and is developing faster with a higher per capita disposable income. Considering the future development plan of the main urban area, it is artificially stipulated that there will be no low-grade elderly care institutions in this urban area. Considering the limited area of land available in the urban areas, it is impossible to increase the number of elderly institutions indefinitely [4]. For this reason, the number of capped elderly institutions is set separately according to the difference in population density and economic development level of each urban area.

Except for the central city, the other sub-centers are at roughly the same level of economic development. To facilitate uniform planning in these sub-centers, we have set the number of high-grade institutions in these areas to be roughly equal.

As an important part of a residential care home, Catering facilities are essential for communication and social interaction among the elderly.

$$
\frac{\sum_{1}^{5} S_{i} a_{i}}{\sum_{1}^{5} A_{i}+\sum_{1}^{5} B_{i}+\sum_{1}^{5} C_{i}+\sum_{1}^{5} D_{i}} \geq S_{0}
$$

Where $S_{i}$ donates the size of catering facilities in level $i$ nursing homes, and $S_{0}$ donates the standard area data 
required by policies. Meanwhile, $\alpha i=A i+B i+C i+D i$, $i=1,2,3,4,5$.

The same applies to active recreational areas, for which we have kept the average communal area of each residential care facility above the minimum required by the comfort standards and the needs of the actual situation.

$$
\frac{\sum_{1}^{5} E_{i} a_{i}}{\sum_{1}^{5} A_{i}+\sum_{1}^{5} B_{i}+\sum_{1}^{5} C_{i}+\sum_{1}^{5} D_{i}} \geq E_{0}
$$

Where $\mathrm{E} i$ donates the size of recreational areas in level i nursing homes, and E 0 donates the standard area data required by policies.

\subsubsection{Health Care}

The number of nurses varies between the different levels of care according to the hierarchy norms. For planning purposes, the number of medical staff: the number of medical and nursing staff in the average area needs to exceed the required number for the corresponding level of urban development.

$$
\frac{\sum_{1}^{5} N_{i} a_{i}}{\sum_{1}^{5} A_{i}+\sum_{1}^{5} B_{i}+\sum_{1}^{5} C_{i}+\sum_{1}^{5} D_{i}} \geq N_{0}
$$

Where $\mathrm{N}$ i donates the number of medical and nursing staff in level i nursing homes, and $\mathrm{N}_{-} 0$ donates the standard number required by policies.

\subsubsection{Summary of Constraints}

$$
\left\{\begin{array}{c}
\beta_{\min } \leq \beta_{i} \leq \beta_{\max } \\
\frac{A_{4}+A_{5}+B_{4}+B_{5}+C_{4}+C_{5}+D_{4}+D_{5}}{\sum_{1}^{5} A_{i}+\sum_{1}^{5} B_{i}+\sum_{1}^{5} C_{i}+\sum_{1}^{5} D_{i}} \geq 5 \% \\
\frac{\sum_{1}^{5} S_{i} a_{i}}{\sum_{1}^{5} A_{i}+\sum_{1}^{5} B_{i}+\sum_{1}^{5} C_{i}+\sum_{1}^{5} D_{i}} \geq S_{0} \\
\frac{\sum_{1}^{5} E_{i} a_{i}}{\sum_{1}^{5} A_{i}+\sum_{1}^{5} B_{i}+\sum_{1}^{5} C_{i}+\sum_{1}^{5} D_{i}} \geq E_{0} \\
\frac{\sum_{1}^{5} N_{i} a_{i}}{\sum_{1}^{5} A_{i}+\sum_{1}^{5} B_{i}+\sum_{1}^{5} C_{i}+\sum_{1}^{5} D_{i}} \geq N_{0}
\end{array}\right.
$$

Where $\alpha i=A i+B i+C i+D i, \beta=A, B, C, D, i=1,2,3,4,5$

\subsubsection{Analysis of growth in pensions, institutional fee levels}

\subsubsection{Pension increases}

Pension income is the income available to the elderly population for discretionary use. It is often used as a measure of the aging population of changes in their standard of living. In economics, it is often approximated by an exponential formula.

$$
C_{i}=\left(1+C_{r}\right)^{i-Y} \times C_{0}
$$

where $C i$ denotes the old-age population pension at year $i$, $\mathrm{Cr}$ denotes the average annual pension growth rate, $\mathrm{CO}$ denotes the initial year pension, and Ydenotes the initial year.

\subsubsection{Levels of fees for elderly care facilities}

By fitting the levels of fees charged by each level of care, it can be concluded that the levels of fees charged by institutions approximately satisfy the following equation.

$$
R_{i}=\left(1+R_{r}\right)^{i-Y} \times R_{0}
$$

where $R i$ denotes the level of fees charged by the nursing facility at year $i, R r$ denotes the average annual fee growth rate, and $R 0$ denotes the price charged in the initial year.

\subsubsection{Objective function}

$$
f(x)=\min \sum_{5}^{1} \alpha_{i} f_{i}
$$

$\alpha i$ denotes the total number of institutions at level $i$ in a city, $\alpha i=A i+B i+C i+D i, f i$ denotes the rate per bed for different levels of institutions, $i=1,2,3,4,5$.

\subsection{Solution Strategy}

The model has many variables and complex parameters. To simplify the calculation process, the figures of the two exponential functions of pensions and fees of elderly institutions are calculated. The forecast data last year is taken every three years of planning as the data used in this planning [7]. In this way, multivariate integer non-linear planning can be transformed into multivariate integer linear planning. For purely linear models, the standard solution method can be used.

\section{Case study}

\subsection{Division of city}

In accordance with the principle of "zoning planning and co-ordination", Nanjing is used as an example for validating the planning model.

As the division of Nanjing's urban areas is too detailed,

\begin{tabular}{|c|c|c|}
\hline & Original district & $\begin{array}{l}\text { New } \\
\text { district }\end{array}$ \\
\hline 1 & Qinhuai,Gulou,Jianye,Xuanwu,Yuhuatai & $\begin{array}{l}\text { Nol. } \\
\text { Main } \\
\text { district }\end{array}$ \\
\hline 2 & Pukou, Luhe & $\begin{array}{l}\text { No2. } \\
\text { Jiangbei } \\
\text { district }\end{array}$ \\
\hline 3 & Jiangning, Qixia & $\begin{array}{l}\text { No3. } \\
\text { Jiangqi } \\
\text { district }\end{array}$ \\
\hline 4 & Lishui, Gaochun & $\begin{array}{l}\text { No4. } \\
\text { Nancheng } \\
\text { district }\end{array}$ \\
\hline
\end{tabular}
and the areas are too trivial, to improve the efficiency of the study, it is proposed to combine the areas with similar economic development levels, population density, and geographical proximity, as shown in Table 1.

TABLE I. The division of Nanjing 


\subsection{Population forecast of Nanjing}

\subsubsection{Results of grey forecasting}

After consolidation and processing, the growth in the number of elderly people by region in Nanjing from 2020 to 2035 can be obtained, as shown in Fig. 1 [8]. It can be seen that the total number and proportion of the elderly population in Nanjing, Jiangsu Province is high and on an upward trend.

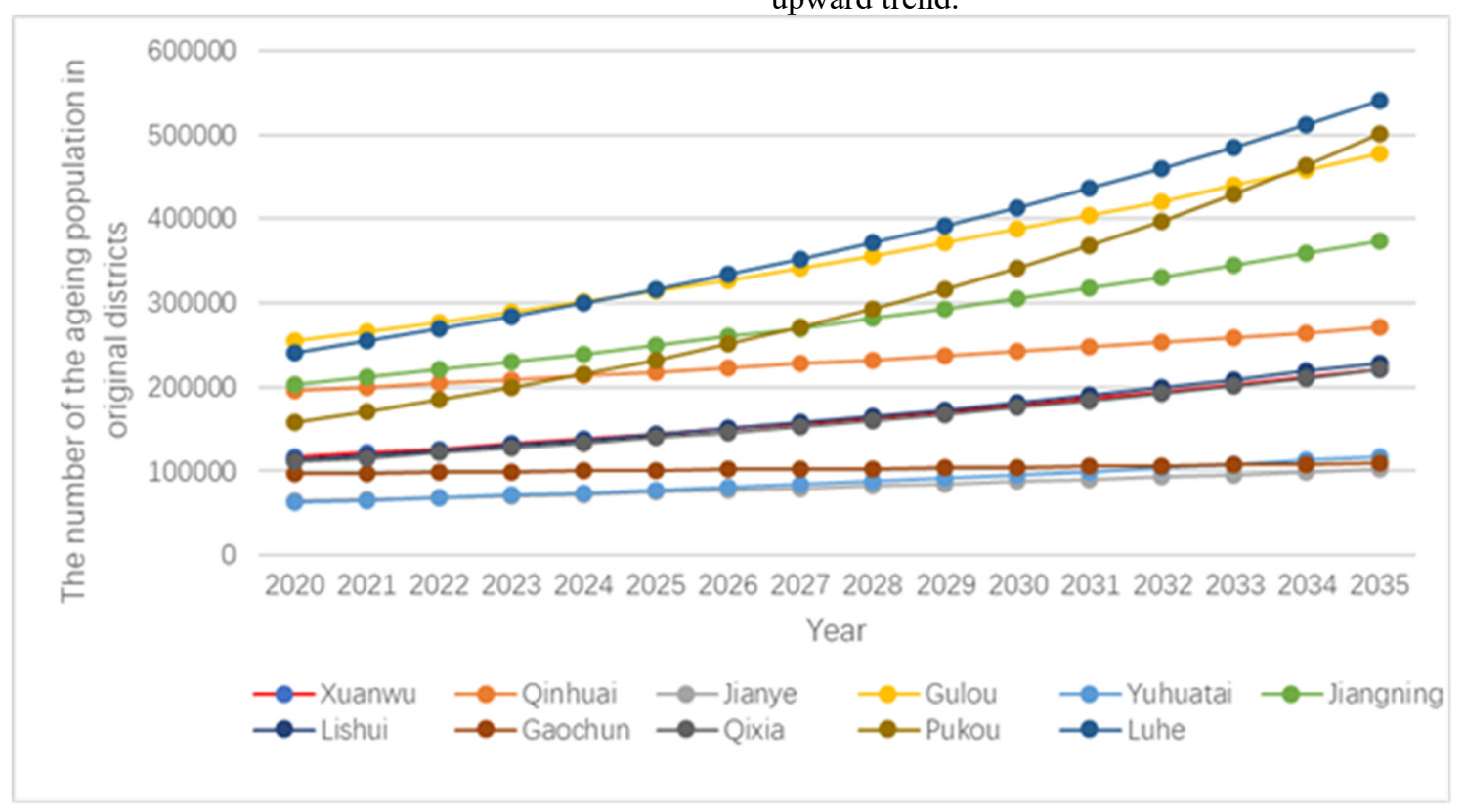

Figure 1. Forecast of the number of the aging population in original districts of Nanjing from 2020 to 2035

\subsubsection{Posterior-Variance-Test}

Taking Gulou District as an example, the probability of small error $\mathrm{P}>0.95$ for most values of the aging population in Gulou District, Nanjing, between 2013 and 2020. The posterior difference ratios $\mathrm{C}$ are all less than 0.45 . That makes the model establishment more scientific and the prediction accuracy grade excellent [9]. Therefore, the prediction results of the model have a high degree of confidence, and the prediction of the aging population in Nanjing, Jiangsu Province, is effective.

\subsubsection{Results of the planning model}

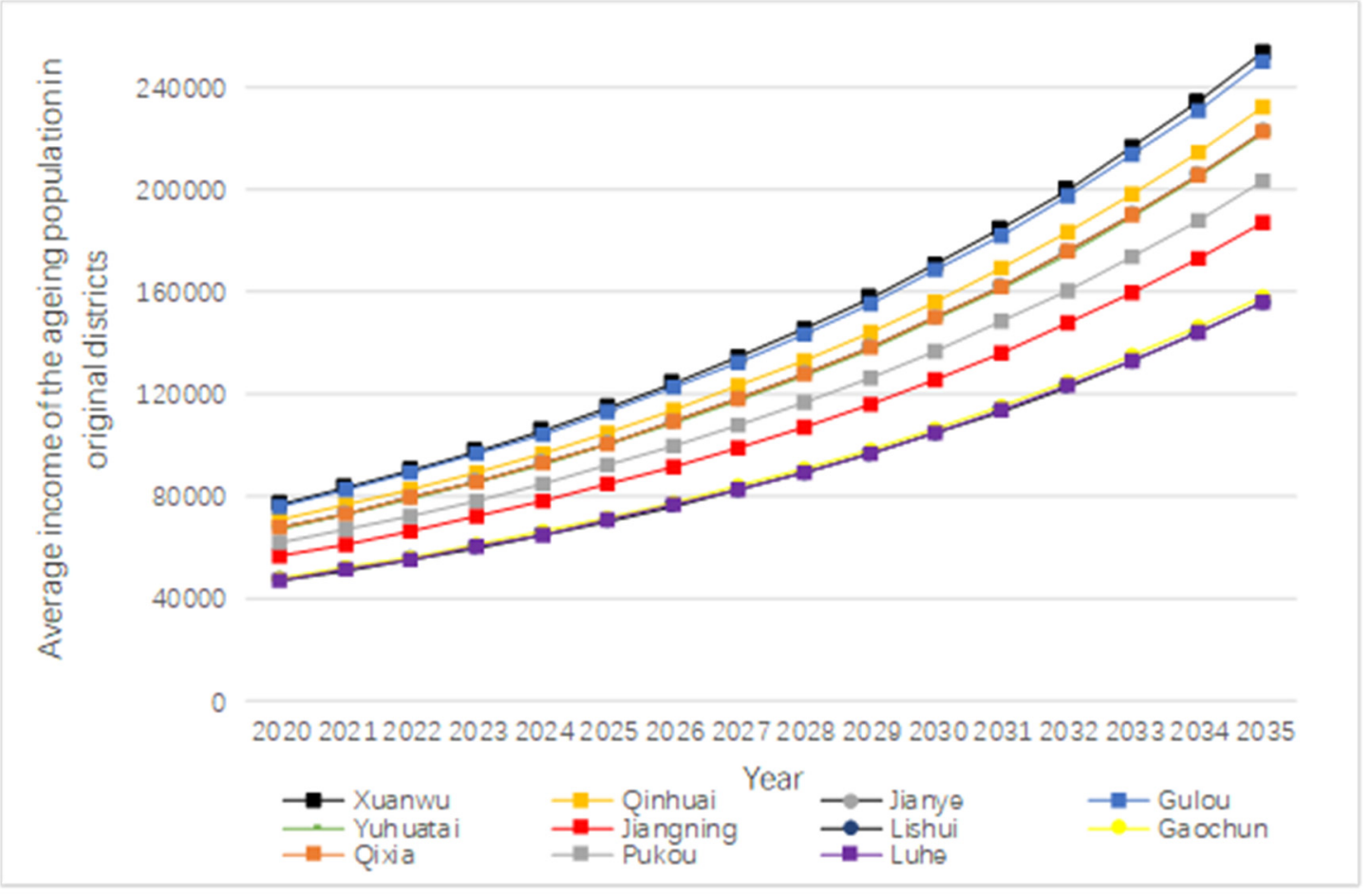

Figure2. The average income of the aging population in original districts 


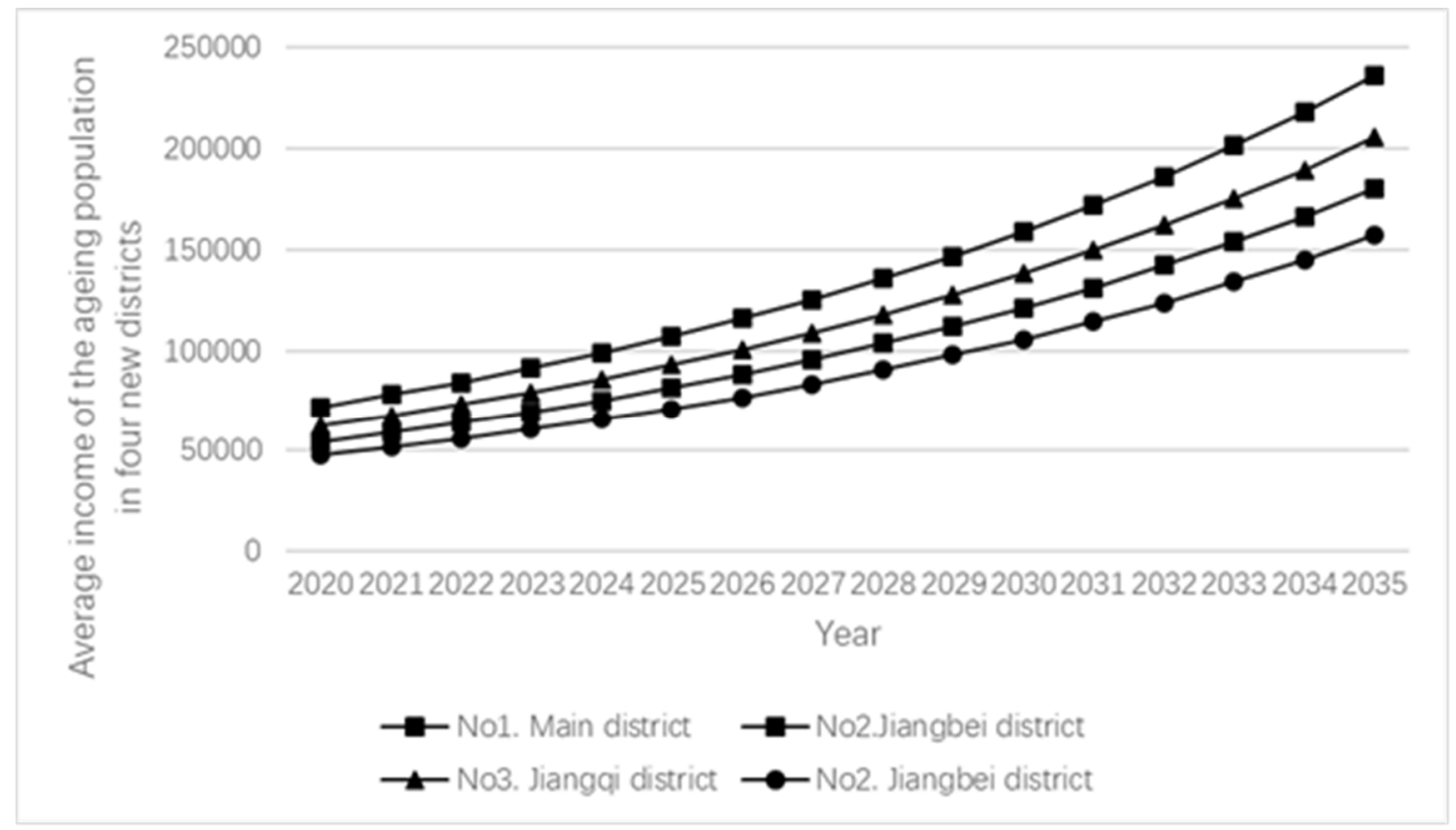

Figure3. The average income of the aging population in new districts

Based on the economic development of Nanjing in recent years, we have calculated an average annual growth rate of $\mathrm{Cr}$ of $8.25 \%$ and a fee level growth rate of $R r$ of $5.3 \%$.

Based on the notice and relevant requirements of the Nanjing Rating Standards for Nursing Homes (for Trial
Implementation), the multivariate integer linear planning results for future nursing homes in Nanjing can be obtained by substituting the data as shown in the table below.

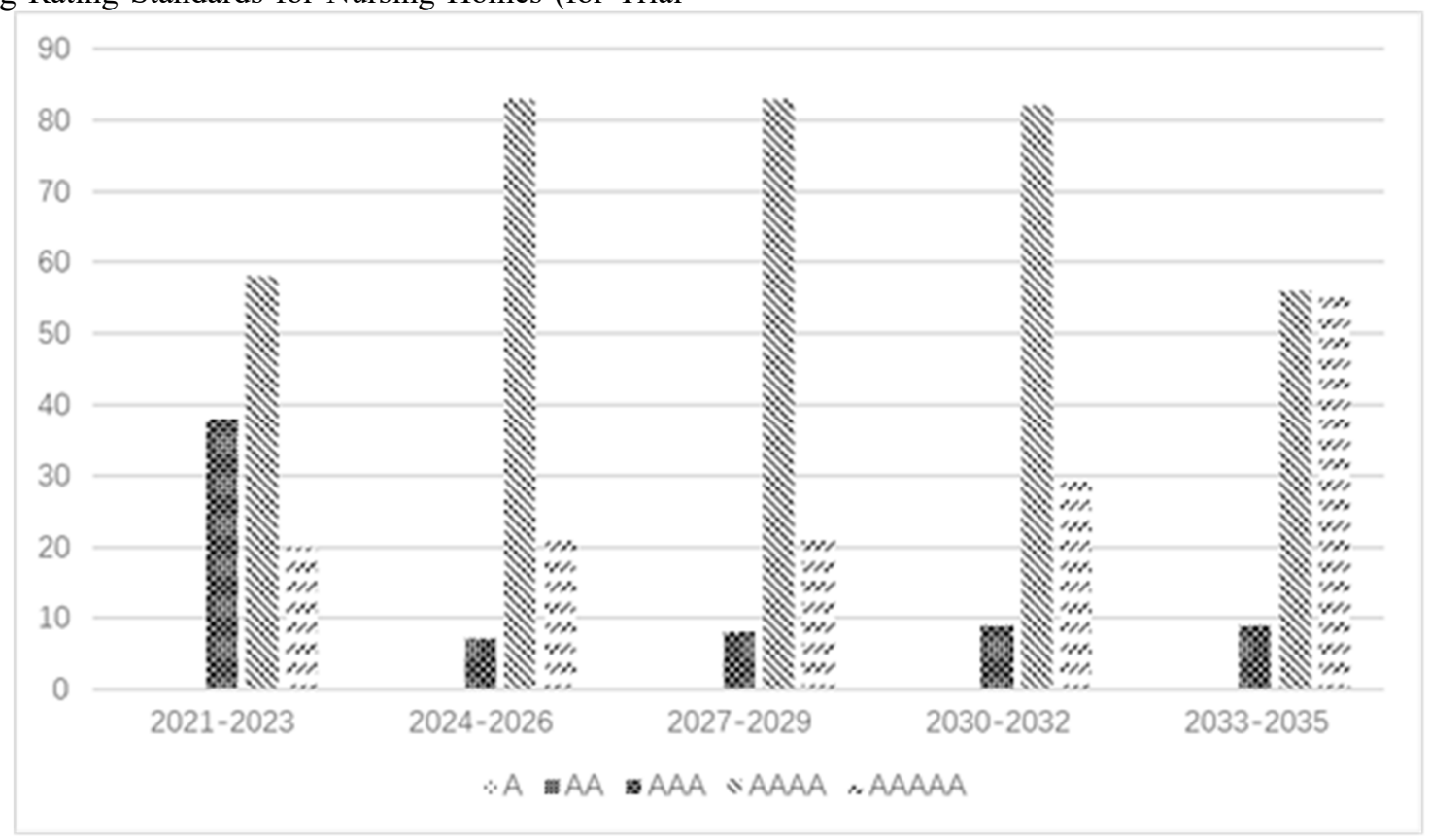

Figure4. The number of proposed elderly care facilities in No1. Main district 


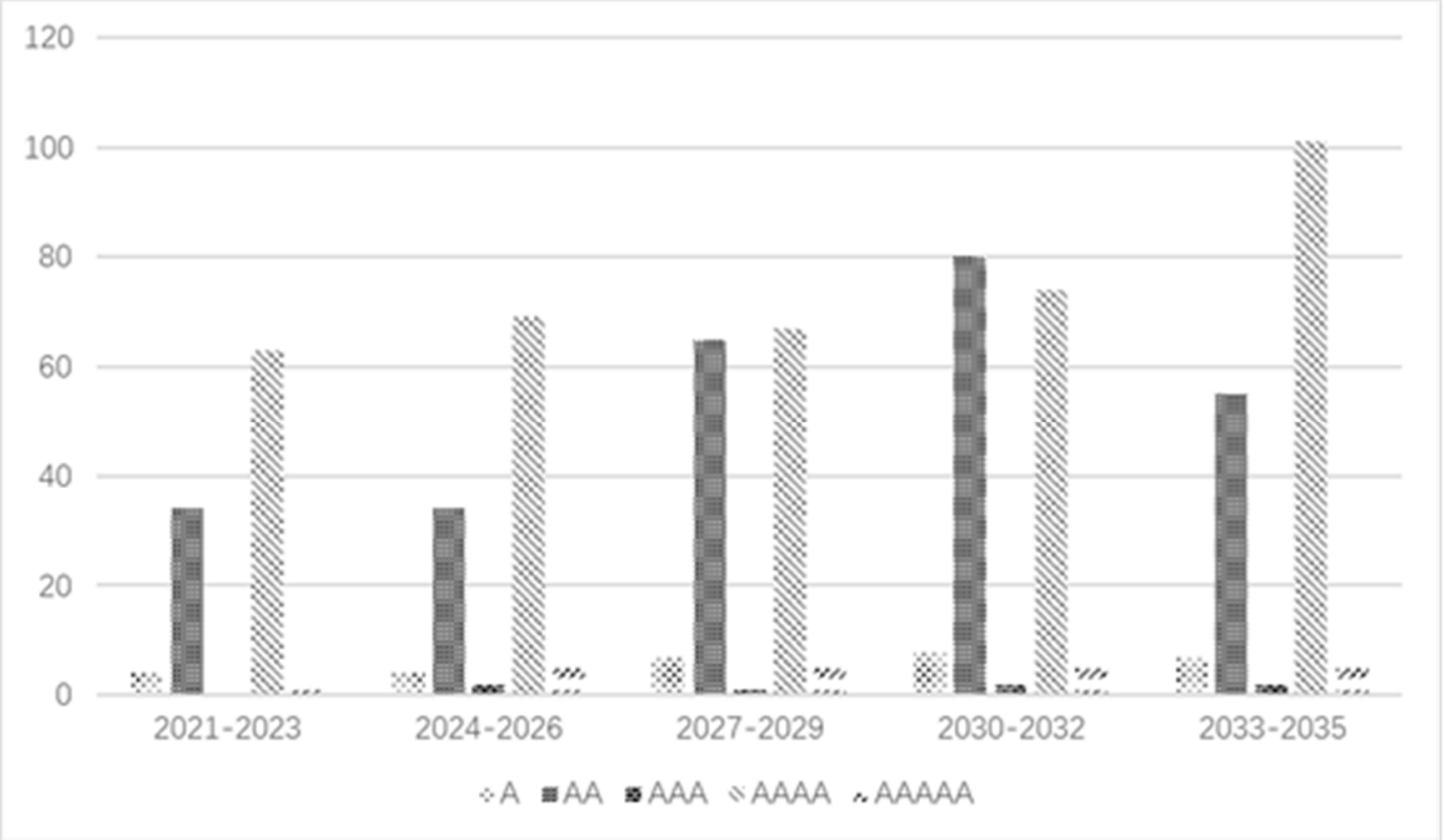

Figure5. The number of proposed elderly care facilities in No2. Jiangbei district

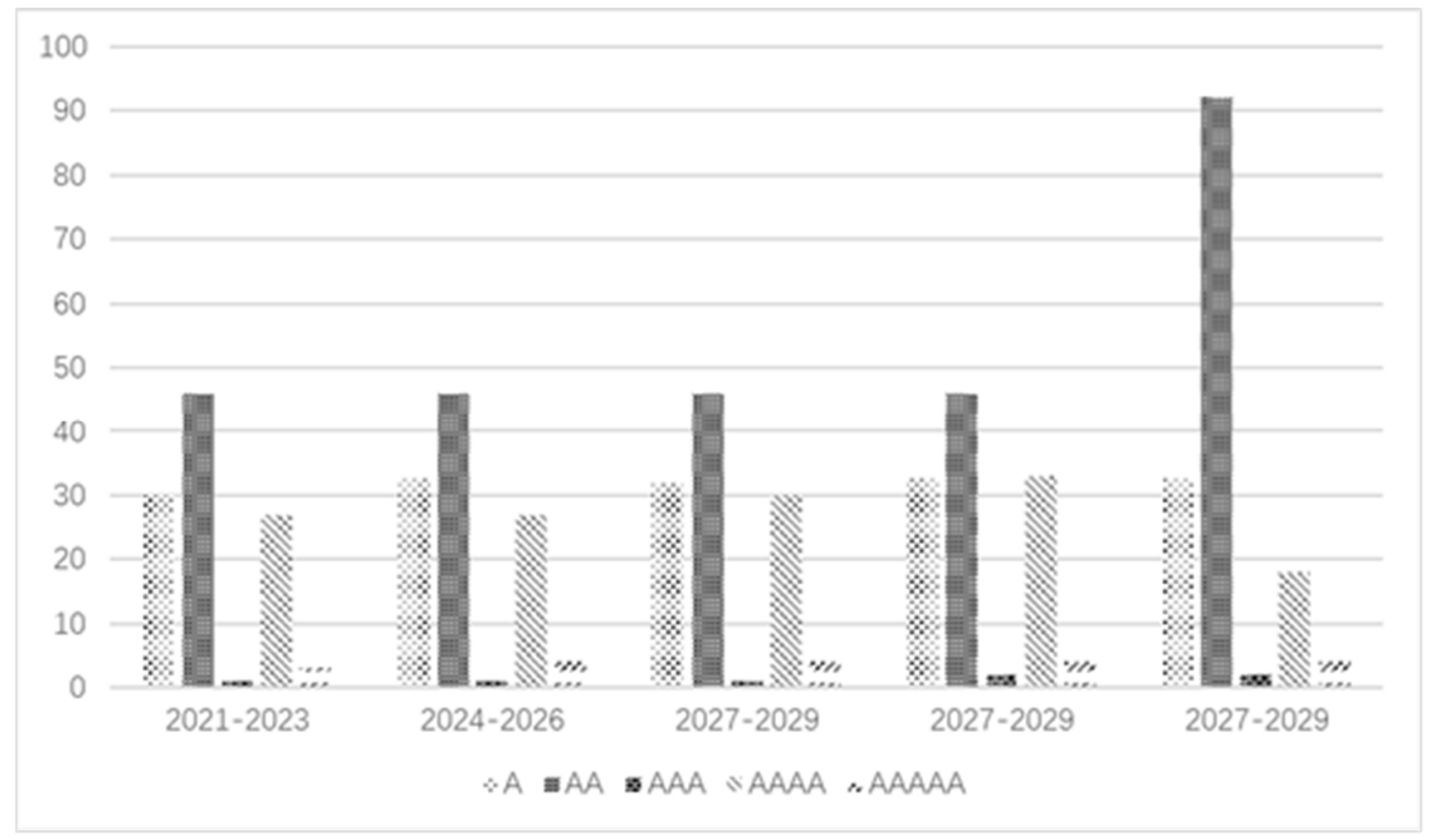

Figure6. The number of proposed elderly care facilities in No3. Jiangqi district 


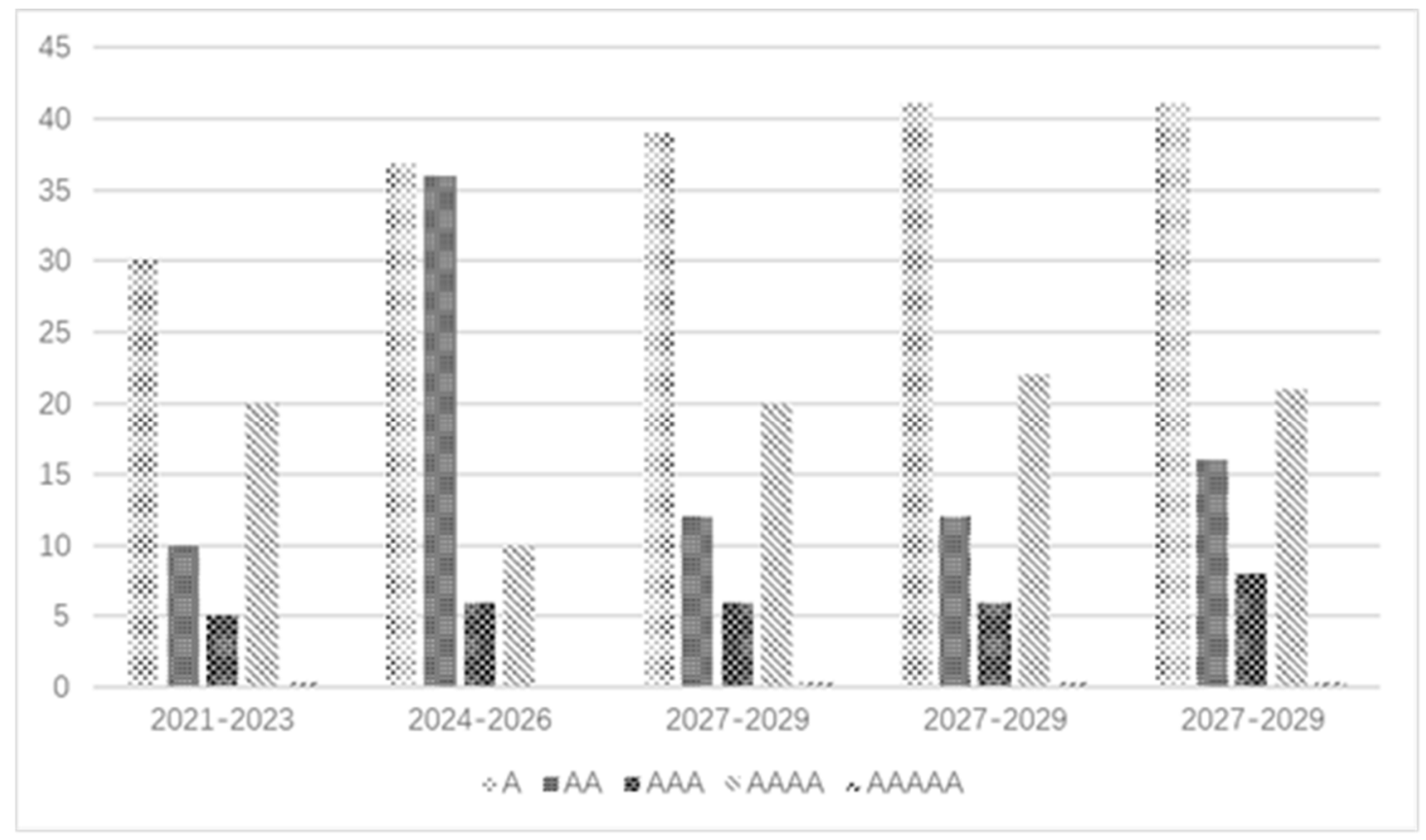

Figure7. The number of proposed elderly care facilities in No4. Nancheng district

\subsubsection{Discussion and analysis}

It can be seen that due to the high per capita wage level and high population density in the main urban area, most of the proposed elderly care institutions are of $4 \mathrm{~A}$ and $5 \mathrm{~A}$ grades. In view of the limited area of the main urban area, $\mathrm{A}$ and 2A grade institutions are not built. Jiangqi District and Nancheng District, due to their relatively large areas and relatively low wage levels, are mostly building lowgrade elderly institutions to meet demand as far as possible. Jiangbei District has a high number of low-grade elderly care institutions due to its relatively dense population. In contrast, many high-grade elderly care institutions have been built in the long-term planning of Jiangbei New District due to the influence of favorable national policies, the rapid rise in wage levels and the steady improvement in people's living standards. Considering the actual topography and economic development level of each region, the higher-grade elderly institutions are placed in the more economically developed and densely populated areas while taking into account that the distribution of each grade of elderly institutions is as equal as possible.

The prediction results of this model are consistent with the policy decisions related to the planning of elderly care institutions in Nanjing, thus verifying the reasonableness of the model.

\section{Conclusion}

In the short term, China's elderly population will continue to grow at a high rate, while at the same time the process of ageing will intensify and the rate of growth of the elderly will increase significantly. During this period, the ageing of our population will enter an unprecedentedly aggressive phase, in terms of scale, speed and depth (ageing). It is therefore necessary to study how to maximise the benefits of elderly resources in an integrated and efficient manner. The model transforms a non-linear problem into a linear one, and the model is relatively simple to solve. Facing different subjects, collecting information about the subjects under study, and understanding the current number, growth trends, and influencing factors of the population to be studied, it is possible to simulate the needs of that population over a certain period and thus make planning. The planning and forecasting model has good universality and can be applied to various spatial scales such as counties, districts, cities, and even provinces. At the same time, the model can be used to forecast not only elderly institutions but also public facilities such as schools, hospitals, and other public facilities. Future scholars could improve this study's zoning method by introducing uninclusive social factors in various ways, effectively improving the accuracy of the zoning and reducing the impact of subjective factors. In addition, in the multivariate linear model section, the effects of the constraints can also be explored in depth, so that the tight constraints of the planning model can be listed and the model can be optimised and simplified at the same time

\section{References}

1. Hu Yuhan, Li Guorui, Zhong Xinyi, Ye Xinchen, Qiu Hengjia. Using Status and Influencing Factors of Home:Based Care Services for The Elderly in Putian City [J]. Northwest Population Journal,2019,40(06):101-113.

2. Wang Zhifeng, Ren Yanlin, Lu Yufeng, Sun Deqiang, Feng Qi. Input-output characteristics and evaluation methods of petroleum engineering science and technology projects [J]. China Petroleum and Chemical Standard and Quality,2018,38(06):140-141. 
3. Li Lu. Prediction and Analysis of Population Aging in Anhui Province_Based on Grey GM $(1,1)$ Model $[\mathrm{J}]$. Journal of Luoyang Institute of Science and Technology (Social Science Edition), 2020, 35(01): $25-31+79$.

4. Chen Jiangyun. Study on the aged care and medical care integrated mode and health security of the elderly under the background of healthy aging[D]. Huazhong University of Science and Technology,2019.

5. Shi Fei,Ju Yang. Towards Sustainable Cities: Using Multiple Linear Regression Model to Identify Influencing Factors to Promote the Use of Public Transport in Downtown Nanjing, China[J]. China City Planning Review,2017,26(04):17-24.

6. Ma Qianqian, Liang Like, Zhang Gaisu, Zhang Jingjing, Li Yang. Research on Spatial Differences of Population Economic Density in Henan Province Based on the County Unit [J]. Journal of Henan University (Natural Science),2017,47(01):1-7+47.

7. Li Peng, Liu Feiyan. Multiple-index Comprehensive Regression Scoring [J]. Journal of Applied Statistics and Management,2014,33(03):408-415.

8. Wang Xiaolong, Yang Guang, Zhang Baohua, Xia Bo, Sun Shouqiang. Application of Gray and Improved Model in Population Forecast [J]. World Sci-Tech R and D,2009,31(04):757-758.

9. Liang Qin. Prediction of Aged Population Based on Grey Prediction Model[D]. Harbin Institute of Technology,2017. 